The Astronomical Journal, 132:1464-1467, 2006 October

(C) 2006. The American Astronomical Society. All rights reserved. Printed in U.S.A.

\title{
XMM-NEWTON DETECTION OF THE RARE FANAROFF-RILEY TYPE II BROAD ABSORPTION LINE QUASAR FIRST J101614.3+520916
}

\author{
Justin J. Schaefer, ${ }^{1,2}$ Michael S. Brotherton, ${ }^{1}$ Zhaohui Shang, ${ }^{1}$ Michael D. GregG, ${ }^{3,4}$ Robert H. Becker, ${ }^{3,4}$ \\ Sally A. Laurent-Muehleisen, ${ }^{3}$ Mark Lacy, ${ }^{5}$ and Richard L. White ${ }^{6}$ \\ Received 2005 July 5; accepted 2006 June 6
}

\begin{abstract}
We have detected FIRST J101614.3+520916 with the XMM-Newton X-ray observatory. FIRST J101614.3+520916, one of the most extreme radio-loud, broad absorption line (BAL) quasars so far discovered, is also a Fanaroff-Riley type II radio source. We find that, compared to its estimated intrinsic X-ray flux, the observed X-rays are likely suppressed and that the observed hardness ratio indicates significant soft X-ray photons. This is inconsistent with the simplest model, a normal quasar spectrum absorbed by a large neutral $\mathrm{H}$ i column density, which would primarily absorb the softer photons. More complex models, involving partial covering, an ionized absorber, ionized mirror reflection, or jet contributions need to be invoked to explain this source. The suppressed but soft X-ray emission in this radioloud BAL quasar is consistent with the behavior displayed by other BAL quasars, both radio-loud and radio-quiet. Key words: quasars: absorption lines — quasars: general — quasars: individual (FIRST J101614.3+520916) — $\mathrm{X}$-rays: galaxies
\end{abstract}

\section{INTRODUCTION}

About $10 \%-20 \%$ of quasars show broad absorption lines (BALs), especially in their UV spectra. These absorption features usually extend to velocities as high as $\sim 10^{4} \mathrm{~km} \mathrm{~s}^{-1}$ relative to the emission lines, indicating high-velocity outflows in the quasars. These absorbers have been identified with winds blowing from an obscuring torus or arising from smaller scales associated with an accretion disk feeding a supermassive black hole. The popular orientation model suggests that BAL quasars are normal quasars viewed along a specific line of sight, or particularly edge-on, skimming the torus or through a wind (e.g., Weymann et al. 1991). Although in this picture quasar radio properties and BALs would seem to be independent, no radio-loud BAL quasars were found for a long time. It remained so until deep radio surveys like the NRAO VLA Sky Survey (NVSS; Condon et al. 1998) and FIRST Bright Quasar Survey (FBQS; Becker et al. 1995; Gregg et al. 1996; White et al. 2000) were conducted, surveying large areas to millijansky levels, and radio-loud BAL quasars started to be identified (Becker et al. 1997, 2000; Brotherton et al. 1998, 2002; Menou et al. 2001). Still, BAL quasar frequency does drop significantly among the most radio-loud quasars (Becker et al. 2001). Becker et al. (2000) studied 27 BAL quasars from the FBQS sample and found that they show a wide range of radio spectral indices, from flat to steep, indicating that a range of orientations is present and therefore strongly challenging the orientation model.

\footnotetext{
${ }^{1}$ Department of Physics and Astronomy, University of Wyoming, Laramie, WY 82072.

${ }^{2}$ Department of Astronomy, University of Florida, 211 Bryant Space Science Center, Gainesville, FL 32611.

${ }^{3}$ Department of Physics, University of California, 1 Shields Avenue, Davis, CA 95616.

${ }^{4}$ Institute of Geophysics and Planetary Physics, Lawrence Livermore National Laboratory, L-413, 7000 East Avenue, Livermore, CA 94550.

${ }^{5}$ Spitzer Science Center, California Institute of Technology, MC 220-6, Pasadena, CA 91125.

${ }^{6}$ Space Telescope Science Institute, 3700 San Martin Drive, Baltimore, MD 21218.
}

So far, only a few radio-loud BAL quasars have been studied at X-ray energies (Brotherton et al. 2005). FIRST J101614.3+ 520916 (hereafter J1016+5209) is the first confirmed BAL quasar that has also been identified as a radio-loud Fanaroff-Riley type II (FR II) source (Gregg et al. 2000). Figure 1 shows the BALs in the rest-frame UV spectrum of J1016+5209, and Table 1 provides its optical and radio parameters. The radio luminosity places it at the extreme end of radio-loud BAL quasars. Its double-lobed radio morphology and luminosity indicate a classic FR II radio source. Gregg et al. (2000) argue that J1016+5209 is a rejuvenated quasar, possibly through a merger or interaction. We note that another known radio-loud FR II BAL quasar is LBQS 1138-0126 (Brotherton et al. 2002) and that the doubleradio-lobed BAL quasar candidate PKS 1004+13 (Wills et al. 1999) has recently been confirmed by Hubble Space Telescope (HST) observation (B. J. Wills et al. 2006, private communication) as a bona fide FR II BAL quasar. The other known radio-loud BAL quasars have compact structures (Becker et al. 2000).

Observations with the Chandra X-Ray Observatory and XMMNewton show that BAL quasars are up to 2 orders of magnitude fainter in X-rays than non-BAL quasars of the same optical brightness (Green et al. 2001; Sabra \& Hamann 2001; Gallagher et al. 2002; Brotherton et al. 2005). Available X-ray spectral analyses of radio-quiet BAL quasars show that they appear to have normal radio-quiet $X$-ray photon indices $(\Gamma \approx 2)$, partially or totally covered by absorbing columns of $N_{\mathrm{H}} \leq 10^{23} \mathrm{~cm}^{-2}$ (e.g., Gallagher et al. 2002). Although the $N_{\mathrm{H}}$ derived from UV absorption lines cannot account for the absorption in the X-ray wavelengths, the UV and X-ray absorbers are probably closely related (Brandt et al. 2000).

Radio-loud quasars are factors of 2-3 times brighter in $\mathrm{X}$-rays than radio-quiet quasars with the same optical magnitude (Brinkmann et al. 2000) and tend to have harder X-ray spectra ( $\Gamma \sim 1.6$; e.g., Reeves \& Turner 2000; Page et al. 2005). These factors may make them particularly suitable for initial exploratory studies of the intrinsic X-ray properties and the properties of the line-of-sight absorbers (Brotherton et al. 2005). We report the results of a short XMM-Newton observation of J1016+5209 in this paper. We detect the object with enough counts to compute 


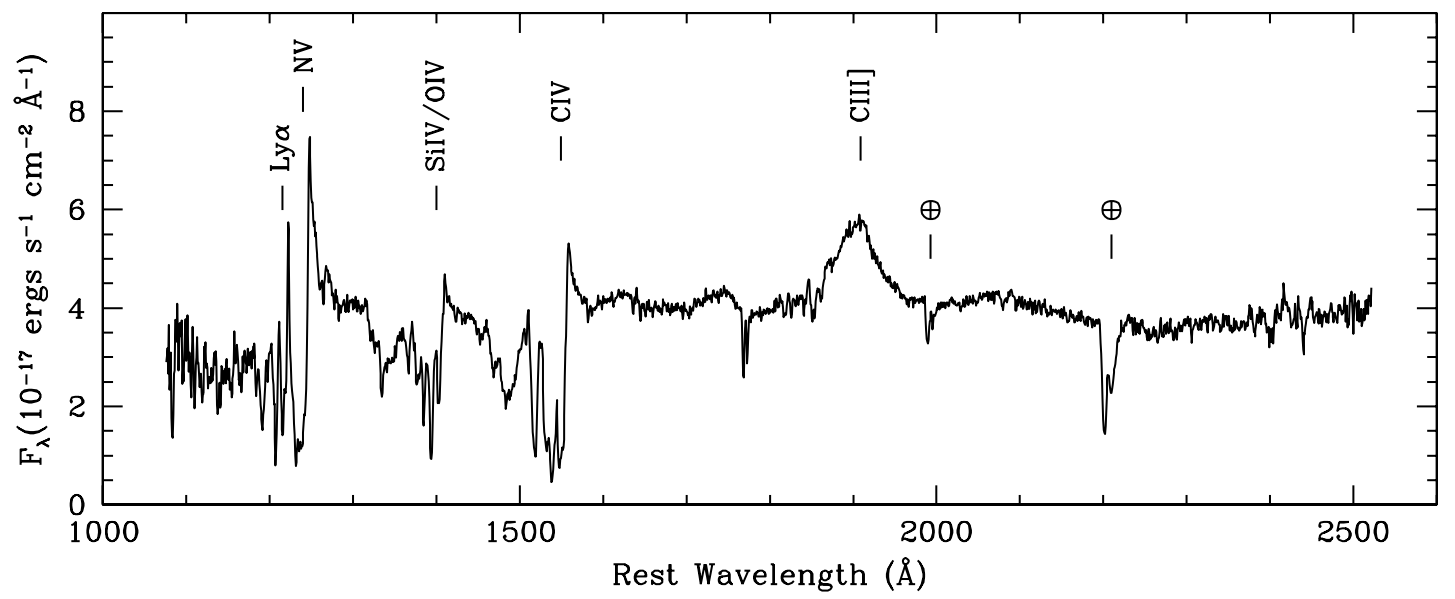

FIG. 1. - Total light spectrum of FIRST J101614.3+520916 from a spectropolarimetric observation obtained with Keck in 2000 January, showing the broad absorption lines. Emission-line positions are marked. Atmospheric absorption bands are also marked with Earth symbols.

a hardness ratio, but not enough for more detailed spectral analysis. Still, the detection can rule out some simple models and has shown us that even the most powerful radio-loud BAL quasars are weak in X-rays.

\section{X-RAY OBSERVATIONS AND DATA ANALYSIS}

We observed FIRST J1016+5209 with XMM-Newton on 2001 November 3 with a duration of $\approx 10 \mathrm{ks}$. This was the first X-ray observation of an FR II BAL quasar. Unfortunately high X-ray background flares limited the usable data of the detectors to only $6.1 \mathrm{ks}$ from the EPIC MOS 1 detector, $5.8 \mathrm{ks}$ from the EPIC MOS 2 detector (Fig. 2), and nothing from the more sensitive EPIC pn detector. Table 2 gives the X-ray properties of FIRST J1016+5209. All of the counts have been background-subtracted. We define the soft X-ray band to be $0.2-2 \mathrm{keV}$ and the hard X-ray band to be $2-8 \mathrm{keV}$. The hardness ratio is then determined to be $\mathrm{HR}=(H-S) /(H+S)=-0.5 \pm 0.08$, where $H$ and $S$ are the source counts in the hard and soft bands, respectively, with errors following Gehrels (1986). Assuming only Galactic absorption, we used PIMMS to estimate that a photon index of 1.76 would give the measured hardness ratio seen in the MOS detectors. There were too few counts (46) for a detailed spectral analysis, but the hardness ratio indicates an excess of soft photons over hard photons.

The $0.2-8 \mathrm{keV}$ flux after Galactic absorption correction, $F_{\mathrm{X}}=$ $6.5 \times 10^{-14} \mathrm{ergs} \mathrm{s}^{-1} \mathrm{~cm}^{-2}$, is calculated using PIMMS and assuming that the average power-law photon index $\Gamma=1.7$ for radio-loud quasars, consistent with our measured HR and those of Reeves \& Turner (2000) and Page et al. (2005). We also estimate the rest-frame optical-X-ray spectral index, $\alpha_{\mathrm{ox}}=-1.06$, using an optical flux at rest frame $2500 \AA$, and an unabsorbed rest-frame $2 \mathrm{keV}$ flux ( $0.579 \mathrm{keV}$ in the observed frame). The unabsorbed rest-frame $2 \mathrm{keV}$ flux was calculated using the observed count rate, PIMMS, and the Galactic absorption, $\Gamma=1.7$, and making a $k$-correction. Cosmological effects in the conversion between fluxes in the observed frame and the rest frame have been taken into account.

While $\alpha_{\mathrm{ox}}=-1.06$ would indicate a rather X-ray-bright BAL quasar, two additional facts should be considered in evaluating the intrinsic X-ray brightness of J1016+5209: the optical flux appears significantly reddened, and the X-ray brightness can also be estimated based on the radio flux.

We estimate the intrinsic X-ray flux of J1016+5209 using the radio-X-ray correlation (Brinkmann et al. 2000) considering the $3 \sigma$ uncertainty about the correlation. ${ }^{7}$ We used the total $5 \mathrm{GHz}$ flux from Gregg et al. (2000) and the relationship shown in Figure 13 of Brinkmann et al. (2000) for radio-loud quasars. The intrinsic X-ray flux is estimated to be $17 \pm 21$ times larger than the observed flux in the ROSAT bandpass. Based on this apparent suppression and an optical flux dereddened for intrinsic reddening (see Gregg et al. 2000), we calculate an intrinsic optical-X-ray spectral index, $\alpha_{\mathrm{ox}}=-1.19$. At $2 \mathrm{keV}$ and $z=2.455$, a neutral H i column density of $N_{\mathrm{H}}=8 \times 10^{23} \mathrm{~cm}^{-2}$ would be required to account for the faintness of the observed $\mathrm{X}$-ray flux. However, such a high $\mathrm{H}$ I column density would result in an extreme hardness ratio, close to unity, inconsistent with our observed $\mathrm{HR}=-0.5$, which is only consistent for a column density of $N_{\mathrm{H}} \leq 1 \times 10^{21.5} \mathrm{~cm}^{-2}$ or less (assuming a normal

\footnotetext{
${ }^{7}$ Due to the scatter in the correlation, the uncertainty in the estimated X-ray flux can be as large as a factor of 1.24. However, this does not affect $\alpha_{\text {ox }}$ very much, because $\alpha_{\text {ox }}$ spans a large frequency range, and an uncertainty of a factor of 2 in X-ray flux only changes $\alpha_{\text {ox }}$ by 0.12 .
}

TABLE 1

Optical and Ratio Properties of J1016+5209

\begin{tabular}{|c|c|c|c|c|c|c|c|c|}
\hline BAL Quasar & $z$ & $\begin{array}{c}E(\approx R) \\
(\mathrm{mag})\end{array}$ & $\begin{array}{l}S_{20 \mathrm{~cm}} \\
(\mathrm{mJy})\end{array}$ & $\begin{array}{c}A_{V}^{\mathrm{a}} \\
(\mathrm{mag})\end{array}$ & $\begin{array}{c}M_{B} \\
(\mathrm{mag})\end{array}$ & $\begin{array}{c}\log \left(L_{5 \mathrm{GHz}}\right) \\
\left(\operatorname{ergs~s}{ }^{-1} \mathrm{~Hz}^{-1}\right)\end{array}$ & $\log \left(R^{*}\right)$ & $\begin{array}{c}f_{2500} \AA^{\mathrm{a}} \\
\left(10^{-29} \mathrm{ergs} \mathrm{s}^{-1} \mathrm{~cm}^{-2} \mathrm{~Hz}^{-1}\right)\end{array}$ \\
\hline $\mathrm{J} 1016+5209$. & 2.455 & 18.6 & 177 & 0.35 & $-26.2(-27.3)$ & 34.3 & $3.4(2.7)$ & $8.24(17.3)$ \\
\hline
\end{tabular}

NoтеS.-Parameters are from Gregg et al. (2000) unless noted. The value $A_{V}$ indicates the intrinsic reddening estimated by the Small Magellanic Cloud reddening law

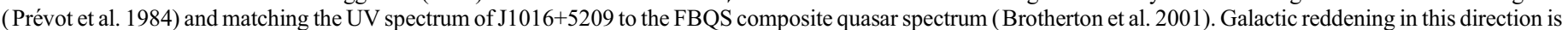

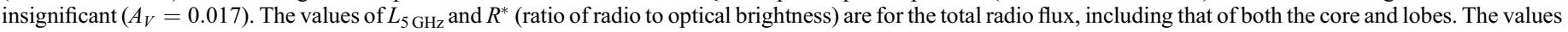

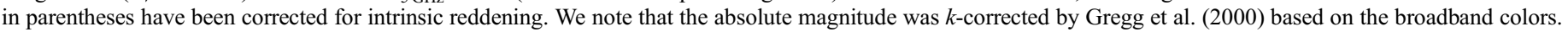

${ }^{a}$ Not from Gregg et al. (2000), but calculated for this work. The values of $f_{2500} \AA$ are in the observed frame. 


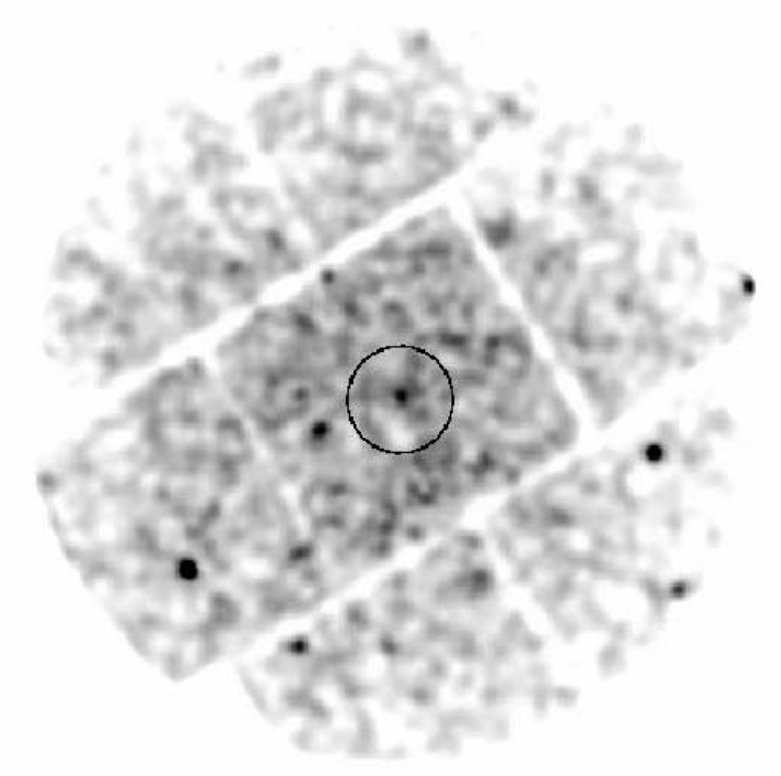

FIG. 2.-FIRST J101614.3+520916, detected by the XMM-Newton MOS2 detector.

radio-loud quasar X-ray slope). In other words, most of the observed soft X-ray photons would have been absorbed if the X-ray source in J1016+5209 were fully covered by such a high column density absorber. Therefore, we conclude that the absorber for $\mathrm{J} 1016+5209$ is not a simple neutral absorber with a high column density. This conclusion should be tempered by the significant uncertainties in these estimates, but it is consistent with what is seen in other BAL quasars.

\section{DISCUSSION}

As mentioned above, the apparently low X-ray flux and the fact that the spectrum is not excessively hard together suggest that a fully covering neutral absorber with a high column density cannot explain our data. Possible alternative scenarios for our observed X-rays include a partially covering neutral absorber, reflection by an ionized mirror, an ionized absorber, or jet contributions.

A partially covering neutral absorber with very high column density would leave the observed X-ray spectrum similar to the incident spectrum except for suppressed X-ray flux. If our estimate of the intrinsic X-ray flux is correct, the covering factor derived from the X-ray reduction factor of 17 for $\mathrm{J} 1016+5209$ would be $94 \%$.

An X-ray spectrum dominated by reflection off an ionized "mirror" (Ross \& Fabian 1993; Ballantyne et al. 2001) could also explain our data, depending on the ionization state of the mirror. In this scenario, at some ionization parameters, $\mathrm{Fe} \mathrm{K} \alpha$ emission would be present in the X-ray spectrum but would require better X-ray observations to be detected.

Ionized absorbers have also often been invoked to explain the X-ray observations of active galactic nuclei (e.g., Kaspi et al. 2002; Grupe et al. 2003; Gallagher et al. 2002, 2004), since these absorbers can also be transparent for soft X-ray photons, but our data set has too few counts to identify any possible absorption edges in order to test this explanation.

Due to the radio-loud nature and lobe-dominated morphology of this object, it is also possible that at least part of the observed $\mathrm{X}$-ray emission is from the jets. Recent high-resolution X-ray observations have made it possible to systematically study X-ray jets and lobes (Sambruna et al. 2002, 2004; Marshall et al. 2005; Croston et al. 2005). The detection rate is typically $\sim 60 \%$ (Sambruna et al. 2004; Marshall et al. 2005). We therefore speculate that X-rays from the accretion disk could be completely absorbed, and we are detecting intrinsically weaker but unabsorbed $\mathrm{X}$-rays from the jets, even if beaming effects are not large given that $\mathrm{J} 1016+5209$ has a steep radio spectrum. The average photon index of the jets is $\sim 1.5$ for a sample of mostly FR II objects (Sambruna et al. 2004), and the core-to-jet X-ray flux ratio has a wide range for the detections in another sample (Marshall et al. 2005), from 5 to about 200. This range covers the suppression factor of this object (17) and most of the radio-loud BAL quasars (42-348) in Brotherton et al. (2005), which have X-ray fluxes consistent with what might be expected arising solely in the jets. Again, better data are required to test this explanation.

Finally, there is a possibility that J1016+5209 is intrinsically $\mathrm{X}$-ray faint or was in a low state at the time of the observation, since some BAL quasars do show significant variability (e.g., Gallagher et al. 2004). Unfortunately our short, high-background observation constitutes more of a detection rather than a light curve, preventing us from detecting variability. However, all BAL quasars so far observed with enough counts for spectral analysis (Gallagher et al. 2001) indicate that X-ray absorption is more likely the primary cause of the "X-ray-weak" quasars (Laor et al. 1997).

Recently, Brotherton et al. (2005) reported X-ray detections of five radio-loud core-dominated BAL quasars with Chandra. The hardness ratio ranges from -0.7 to 0.1 , and $\alpha_{\text {ox }}$ from -0.8 to -2.0 . All five objects also show significant X-ray suppression compared to estimates of their intrinsic X-ray flux. Compared with this sample, J1016+5209 does not seem to be abnormal in $\alpha_{\text {ox }}$ or hardness ratio; our XMM-Newton hardness ratio from Table $2(\mathrm{HR}=-0.5)$ is equivalent to a Chandra $\mathrm{HR}=-0.7$ (estimated using PIMMS, set to CXO3). The X-ray properties of these radio-loud BAL quasars are in general agreement with the results for radio-quiet BAL quasars. Based on their X-ray

TABLE 2

X-Ray Properties of J1016+5209

\begin{tabular}{|c|c|c|c|c|c|c|c|c|}
\hline $\begin{array}{c}\text { BAL Quasar } \\
\text { (1) }\end{array}$ & $\begin{array}{c}N_{\mathrm{H}} \\
\left(\mathrm{cm}^{-2}\right) \\
(2)\end{array}$ & $\begin{array}{c}\text { Rate } \\
\left(10^{-4} \text { counts } s^{-1}\right) \\
(3)\end{array}$ & $\begin{array}{c}\text { Soft } \\
\text { (counts) } \\
(4)\end{array}$ & $\begin{array}{c}\text { Hard } \\
\text { (counts) } \\
(5)\end{array}$ & $\begin{array}{c}S+H \\
\text { (counts) } \\
(6)\end{array}$ & $\begin{array}{l}\mathrm{HR} \\
(7)\end{array}$ & $\begin{array}{c}F_{\mathrm{X}} \\
\left(\operatorname{ergs~s}^{-1} \mathrm{~cm}^{-2}\right) \\
(8)\end{array}$ & $\begin{array}{l}\alpha_{\mathrm{ox}} \\
(9)\end{array}$ \\
\hline 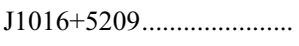 & $7.64 \times 10^{19}$ & $77 \pm 6$ & $36 \pm 2.4$ & $10 \pm 1.3$ & $46 \pm 3.6$ & $-0.5 \pm 0.08$ & $(6.5 \pm 0.7) \times 10^{-14}$ & $-1.06(-1.19)$ \\
\hline
\end{tabular}

Notes.-Col. (2): Galactic neutral hydrogen column density (Dickey \& Lockman 1990). Col. (3): X-ray counts s ${ }^{-1}$ (0.2-8 keV) from the two MOS detectors.

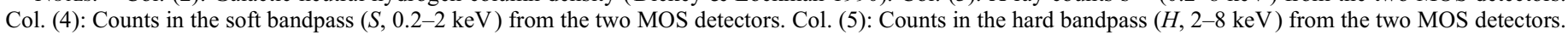

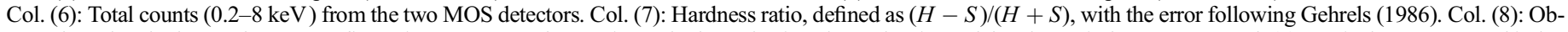

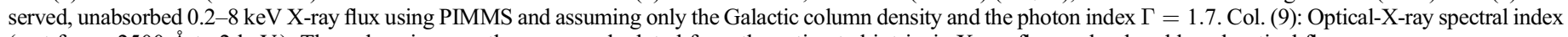
(rest frame $2500 \AA$ to $2 \mathrm{keV}$ ). The values in parentheses are calculated from the estimated intrinsic X-ray flux and a dereddened optical flux. 
spectral analyses, Gallagher et al. (2002) suggested that radioquiet BAL quasars have the typical intrinsic power-law X-ray continuum of normal radio-quiet quasars but with significant $\mathrm{ab}-$ sorption column density. However, they argue that the absorption is likely very complicated, and it is typically not possible to distinguish between a partially covering and an ionized absorber with their data. Grupe et al. (2003) showed excess soft X-ray photons in their spectra of two radio-quiet BAL quasars and also reported that both a partially covering absorber and an ionized absorber could fit their observed spectra. It is still not clear whether radio-loud BAL quasars also have the typical intrinsic $\mathrm{X}$-ray continuum of normal radio-loud quasars or whether the FR II BAL quasars like J1016+5209 have special X-ray properties. High-quality X-ray spectra are needed to answer these questions and to reveal the real X-ray nature of J1016+5209 and other radio-loud BAL quasars.

\section{CONCLUSIONS}

We have observed and detected the first confirmed radio-loud FR II BAL quasar, FIRST J1016+5209, in the X-ray with XMMNewton. We have enough counts to derive the hardness ratio but not enough for detailed spectral analysis. The X-ray flux appears to be suppressed by a factor of 17 relative to the intrinsic X-rays estimated from the radio-X-ray correlation, although significant uncertainties are associated with this factor. If the X-rays were suppressed due to absorption associated with a high column density of neutral hydrogen, the X-rays observed would be much harder, which is inconsistent with the observations. This implies that the X-ray absorption in J1016+5209 is more complicated, such as being from an ionized absorber, an ionized mirror, or something neutral but partially covering the X-ray source. Contributions from a jet are also possible. High-quality X-ray spectra are necessary to understand the nature of the absorber.

This work is funded by the Wyoming NASA Space Grant Consortium, NASA grant NGT-40102, and Wyoming NASA EPSCoR grant NCC5-578. This work is also funded in part by NASA through the US XMM-Newton Program, with data provided by ESA. This work was partly performed under the auspices of the US Department of Energy by the University of California, Lawrence Livermore National Laboratory, under contract W-7405-Eng-48.

\section{REFERENCES}

Ballantyne, D. R., Iwasawa, K., \& Fabian, A. C. 2001, MNRAS, 323, 506

Becker, R. H., Gregg, M. D., Hook, I. M., McMahon, R. G., White, R. L., \& Helfand, D. J. 1997, ApJ, 479, L93

Becker, R. H., White, R. L., Gregg, M. D., Brotherton, M. S., LaurentMuehleisen, S. A., \& Arav, N. 2000, ApJ, 538, 72

Becker, R. H., White, R. L., \& Helfand, D. J. 1995, ApJ, 450, 559

Becker, R. H., et al. 2001, ApJS, 135, 227

Brandt, W. N., Laor, A., \& Wills, B. J. 2000, ApJ, 528, 637

Brinkmann, W., Laurent-Muehleisen, S., Voges, W., Siebert, J., Becker, R., Brotherton, M., White, R., \& Gregg, M. 2000, A\&A, 356, 445

Brotherton, M., Croom, S., De Breuck, C., Becker, R., \& Gregg, M. 2002, AJ, 124,2575

Brotherton, M., Laurent-Muehleisen, S. A., Becker, R. H., Gregg, M. D., Telis, G., \& White, R. L. 2005, AJ, 130, 2006

Brotherton, M. S., Tran, H. D., Becker, R. H., Gregg, M. D., Laurent-Muehleisen, S. A., \& White, R. L. 2001, ApJ, 546, 775

Brotherton, M. S., van Breugel, W., Smith, R. J., Boyle, B. J., Shanks, T., Croom, S. M., Miller, L., \& Becker, R. H. 1998, ApJ, 505, L7

Condon, J. J., Cotton, W. D., Greisen, E. W., Yin, Q. F., Perley, R. A., Taylor, G. B., \& Broderick, J. J. 1998, AJ, 115, 1693

Croston, J. H., Hardcastle, M. J., Harris, D. E., Belsole, E., Birkinshaw, M., \& Worrall, D. M. 2005, ApJ, 626, 733

Dickey, J., \& Lockman, F. 1990, ARA\&A, 28, 215

Gallagher, S., Brandt, W. N., Chartas, G., \& Garmire, G. 2002, ApJ, 567, 37

Gallagher, S. C., Brandt, W. N., Laor, A., Elvis, M., Mathur, S., Wills, B. J., \& Iyomoto, N. 2001, ApJ, 546, 795

Gallagher, S. C., Brandt, W. N., Wills, B. J., Charlton, J. C., Chartas, G., \& Laor, A. 2004, ApJ, 603, 425
Gehrels, N. 1986, ApJ, 303, 336

Green, P., Aldcroft, T., Mathur, S., Wilkes, B., \& Elvis, M. 2001, ApJ, 558, 109 Gregg, M., Becker, R., Brotherton, M., Laurent-Muehleisen, S., Lacy, M., \& White, R. 2000, ApJ, 544, 142

Gregg, M., Becker, R. H., White, R. L., Helfand, D. J., McMahon, R. G., \& Hook, I. M. 1996, AJ, 112, 407

Grupe, D., Mathur, S., \& Elvis, M. 2003, AJ, 126, 1159

Kaspi, S., et al. 2002, ApJ, 574, 643

Laor, A., Fiore, F., Elvis, M., Wilkes, B. J., \& McDowell, J. C. 1997, ApJ, 477, 93

Marshall, H. L., et al. 2005, ApJS, 156, 13

Menou, K., et al. 2001, ApJ, 561, 645

Page, K. L., Reeves, J. N., O’Brien, P. T., \& Turner, M. J. L. 2005, MNRAS, 364, 195

Prévot, M. L., Lequeux, J., Prévot, L., Maurice, E., \& Rocca-Volmerange, B. 1984, A\&A, 132, 389

Reeves, J. N., \& Turner, M. J. L. 2000, MNRAS, 316, 234

Ross, R. R., \& Fabian, A. C. 1993, MNRAS, 261, 74

Sabra, B. M., \& Hamann, F. 2001, ApJ, 563, 555

Sambruna, R. M., Gambill, J. K., Maraschi, L., Tavecchio, F., Cerutti, R., Cheung, C. C., Urry, C. M., \& Chartas, G. 2004, ApJ, 608, 698

Sambruna, R. M., Maraschi, L., Tavecchio, F., Urry, C. M., Cheung, C. C., Chartas, G., Scarpa, R., \& Gambill, J. K. 2002, ApJ, 571, 206

Weymann, R. J., Morris, S. L., Foltz, C. B., \& Hewett, P. C. 1991, ApJ, 373, 23

White, R. L., et al. 2000, ApJS, 126, 133

Wills, B. J., Brandt, W. N., \& Laor, A. 1999, ApJ, 520, L91 